\title{
FLUX LOOPS: MORE NEW HEAVY PARTICLES IN THE WEINBERG-SALAM MODEL
}

\author{
Martin B. EINHORN \\ University of Michigan, Department of Physics, Ann Arbor, MI 48109, USA \\ and \\ Robert SAVIT \\ Fermi National Accelerator Laboratory, Batavia, IL 60510, USA
}

Received 10 May 1978

\begin{abstract}
Extending Nambu's suggestion that there are stringlike classical solutions in the Weinberg-Salam model, we argue that these include closed tori of weak neutral $\left(\mathrm{Z}^{0}\right)$ flux.
\end{abstract}

In an insightful discussion, Nambu [1] has suggested that the spectrum of the Weinberg-Salam model may be even richer than previously expected. He argues that there are very likely classical dumbbell-like solutions of the equations of motion describing a monopole-antimonopole pair connected by a stringlike tube of neutral weak $\left(Z^{0}\right)$ flux. These solutions presumably correspond in the quantum theory to a spectrum of narrow resonances, with masses in the range of a few $\mathrm{TeV}$ and with widths characteristic of electromagnetic-weak decays at these energies.

In this note, we extend Nambu's considerations and suggest that the stringlike tubes of flux form closed tori which also generate a spectrum of narrow resonances in the $\mathrm{TeV}$ range. Unlike Nambu's rotating dumbbells (magnetic dipoles), which even classically radiate electromagnetically, the closed loops discussed here do not. They may well be completely stable classically (true solitons), although this cannot be determined without a more careful analysis of the classical field equations. Like the dumbbells, the tori give rise to Regge trajectories, but the slope of the leading closed-loop trajectory is less than half the slope of the leading dumbbell trajectory ${ }^{\ddagger 1}$.

Nearly all the general features of our solutions can be inferred from essentially dimensional analysis,

\footnotetext{
Supported in part by the Department of Energy.
}

which we now present. (Later we shall discuss some more technical details.) First let us recall some properties of the weak flux tube [1]. It has a radius $\rho_{0}$ and mass per unit length $\tau_{0}$ given by

$\rho_{0}=2\left(\frac{\cos \theta_{\mathrm{W}}}{m_{\mathrm{H}} m_{\mathrm{W}}}\right)^{1 / 2}, \quad \tau_{0}=\frac{\cos \theta_{\mathrm{W}} \sin ^{2} \theta_{\mathrm{W}} m_{\mathrm{H}} m_{\mathrm{W}}}{\alpha}$,

where $\theta_{\mathrm{W}}$ is the Weinberg angle, $m_{\mathrm{W}}$ the mass of the charged weak vector boson, $m_{\mathrm{H}}$ the mass of the Higgs scalar meson. As usual, $\alpha=e^{2 / 4 \pi} \approx 1 / 137$. Suppose we have a closed loop of flux. To justify the string ap. proximation, the length of the loop $L$ must be large compared to the diameter of the flux tube, $L \gg 2 \rho^{0}$. If the loop were static, it would have a mass $L \tau_{0}$ $\gg 2 \rho^{0} \tau^{0}$; in motion, it will be somewhat larger. Now we have as a characteristic mass scale for the flux loop the quantity:

$\rho_{0} \tau_{0}=\frac{2 \cos ^{3 / 2} \theta_{\mathrm{W}} \sin ^{2} \theta_{\mathrm{W}}}{\alpha} \sqrt{m_{\mathrm{H}} m_{\mathrm{W}}}$.

₹1 All these properties are, of course, in close analogy with the dual resonance model of hadrons. (For reviews, see Jacob [2] and Scherk [2].) If the monopoles are analogous to quarks and the flux tube analogous to the dual string, then the dumbbells are like ordinary mesons while the tori are like quarkless mesons (in QCD, the latter are associated with glueballs, mesons of purely gluonic origin). 
In estimating its magnitude, our greatest uncertainty is the Higgs mass. To be optimistic, we will choose 5 $\mathrm{GeV}$, and leave the reader to correct for his/her own favorite value. Then for $\sin ^{2} \theta_{\mathrm{W}}=1 / 4\left(m_{\mathrm{W}}=75 \mathrm{GeV}\right)$, we find $\rho_{0} \approx 0.02 \mathrm{fm}, \rho_{0} \tau_{0} \approx 1 \mathrm{TeV}$.

Since the string approximation requires that the size of the loop be large compared to the radius of the flux tube, we conclude that several $\mathrm{TeV}$ is a lower limit for a classical, stringlike torus.

As with dumbbells [1] , the torus must be dynamically stabilized by some sort of periodic motion, a rotation or oscillation. The general solution for the motion of the classical, relativistic, closed string may be summarized [2] as follows: Let $\boldsymbol{x}(\tau, \sigma)$ be the coordinates of the curve described by the string. The parameter $\tau$ will be taken proportional to the time, $t=2 \alpha^{\prime} M \tau$, where the "Regge slope" $\alpha$ ' is related to the string tension according to $\alpha^{\prime}=1 / 2 \pi \tau_{0}$. ( $M$ is the invariant mass of the string, i.e., its total energy in the center-ofmass frame.) The parameter $\sigma \in[0,2 \pi]$ describes the curve at a fixed time. Because the string is closed, $\boldsymbol{x}(\tau, 0)=\boldsymbol{x}(\tau, 2 \pi)$. In the center-of-mass frame, the general solution may be written as

$\boldsymbol{x}=\sum_{n \neq 0}\left(\boldsymbol{A}_{n} \mathrm{e}^{\mathrm{i} n \sigma}+\boldsymbol{B}_{n} \mathrm{e}^{-\mathrm{i} n \sigma}\right) \mathrm{e}^{-\mathrm{i} n \tau}$,

where the constants of the motion $\boldsymbol{A}_{n}, \boldsymbol{B}_{n}$ are subject to the following constraints: $\boldsymbol{A}_{-n}=\boldsymbol{A}_{n}^{*}$ (reality), $\boldsymbol{B}_{-n}$ $=\boldsymbol{B}_{n}^{*}$.

Define

$L_{k}^{(A)} \equiv \sum_{n} n(k-n) A_{n} \cdot A_{k-n}$,

$L_{k}^{(B)} \equiv \sum_{n} n(k-n) \boldsymbol{B}_{n} \cdot \boldsymbol{B}_{k-n}$.

Then $L_{k}^{(A)}=L_{k}^{(B)}=0$ for $k \neq 0$ (Virasovo conditions) and $L_{0}^{(A)}=L_{0}^{(B)}=-\left(\alpha^{\prime} M\right)^{2}$.

What are some simple examples of such solutions? The canonical solution in discussions of dual models [2] is a loop tightly drawn along a line, tumbling about its center (fig. 1a), a motion on the leading trajectory. In the context of a stringlike approximation to a classical solution of the field theory, this is evidently unacceptable since it corresponds to overlapping flux tubes. Another possible simple motion is described by a circle whose radius oscillates between some maximum value and zero (fig. $1 \mathrm{~b}$ ). This is ap-

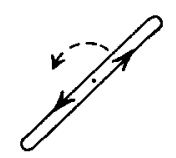

(1a)

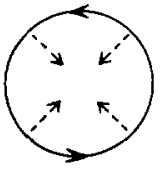

(1b)
Fig. 1. Two simple, single frequency classical motions. (a) Leading Regge trajectory, (b) a motion having zero angular momentum.

pealing because it has angular momentum zero, but here again the string approximation breaks down. When the radius of the circle is on the order of the diameter $2 \rho_{0}$ of the flux tube, it is not sensible to speak of a well-defined string. (Note, however, there could well be classical solutions of the field theory roughly of this type. See below.) It is unfortunately not easy to find simple motions for which the string approximation is valid at all times. One can show that every motion consisting of a single frequency $N$ (i.e., $\boldsymbol{A}_{n}=\boldsymbol{B}_{n}=0$ for all $n \neq N$ ) has the property that the string overlaps itself at some time. It is possible that inclusion of other eigenfrequencies could circumvent this conclusion. Nevertheless, we believe that there are very likely classically stable, monopole-free motions of pure $Z^{0}$-flux which resemble closed tori at least much of the time.

We turn to discuss some interesting questions raised by our investigation. First, it is important to determine whether lower mass, classical solutions exist even though the string approximation to the flux tube or the point mass approximation to the monopole is invalid. If such states lie on approximately linear Regge trajectories which extend down to low spin states, then it can easily be estimated that the low angular momentum states will have masses an order of magnitude smaller than those found here, i.e., in the "low mass" region of perhaps hundreds of GeV. These are not so very much heavier than the elementary vector bosons themselves. Secondly, the stability of any of the classical solutions remains to be shown.

It is interesting to ask how any of the soliton states we have been discussing would decay. Nambu's dumbbells are not even stable classically [1] and lose energy due to electromagnetic radiation. Our tori might be stable classically. Quantum mechanically, they would 
presumably decay via creation of various particles, such as through the emission of Higgs bosons, elementary vector bosons (especially $Z^{0}$ ), and lepton-antilepton or quark-antiquark pairs. (Dumbbells would also participate in some of these mechanisms.) Given the classical soliton solutions of the field equations, how could one in principle, calculate such effects? The propagator for a soliton may be represented by the usual path integral expression by requiring that the quantum fields tend asymptotically to the classical configurations [3]. The width of the state may be inferred from the imaginary part of the inverse propagator. Such a procedure might even be implementable in a weak-coupling theory such as this. A specific decay amplitude could be determined from an overlap path integral between an incoming soliton and a given outgoing state, such as a fermion-antifermion pair.

As Nambu illustrated [1], these kinds of solitons are sensitive to both the gauge group and the particular representation of Higgs fields. Consequently, it should be very interesting to investigate other popular unified gauge theories for similar states. Unfortunately, it may be difficult to find such solutions in general. Because they are not completely topologically stable, it is not possible to infer their existence from the usual homotopy arguments [4]. We do not know of a systematic procedure for discovering these solutions, but we will make a couple of remarks. First, it is worth noting that these solutions are not without topological significance. The stability of the flux tube, for example, stems from the existence of a topological conservation law in two space dimensions, viz., flux quantization or vorticity. Consequently, one general approach to discovering solutions of the type found by Nambu and by us would be to use homotopic methods in two dimensions to determine the properties of tubelike solutions and then dy namically stabilize three dimensional solutions as outlined here. So long as the characteristic size of the three dimensional object is large compared to the diameter of the tube, this should be a good approximation. Another similar approach suggested by Nambu's construction is to relax the finite energy requirement and explore the asymptotic differential equations, possibly by topological methods. This is essentially the method he used to find the semi-infinite flux tube terminating on a monopole.

A third method might be to draw analogies with similar lattice gauge theories. When the electromagnetic field $A^{\mu}$ and the charged vector boson fields $\mathrm{W}_{+}^{\mu}$ are all set equal to zero, the resulting lagrangian involving only the $Z^{0}$ vector field is identical to the abelian Higgs model. Indeed, the "topological" excitations of this $U(1)$ theory on a lattice are precisely of the dumbbell and closed-loop types [5] ${ }^{\ddagger 2}$ although, it must be noted, the origin of the monopoles is quite different. In addition, the stability of these structures is provided by the rigidity of the lattice, in contrast to the continuum theory where rotations or oscillations may be required for stability in the absence of a short distance cut-off.

It is worth noting that certain types of non-topological solitons have been found by variational methods [6] . However, these generally exploit a conserved "charge" related to some underlying symmetry by Noether's theorem. Consequently, these methods would not appear to be transferable to the types of solutions under discussion here ${ }^{\ddagger 3}$.

As noted earlier, Nambu's solutions are not even stable at the classical level, which renders the problem of finding a general approach even more challenging. How should one search for an unstable solution to the classical field equations whose ratio of width to mass happens to be small?

In summary it seems likely that the spectrum of particles in the Weinberg-Salam model and certain other unified theories includes new heavy particle states in the range of hundreds of $\mathrm{GeV}$ up to many $\mathrm{TeV}$. An examination of their general properties (including their production and decay mechanisms) and an investigation of the theoretical questions raised above are important for understanding the role of these intriguing states.

\$2 It should be noted however that the origin of monopoles in the two cases is quite different: in the abelian Higgs model on a lattice, they arise from the compact $U(1)$ group; in the Weinberg-Salam model, they are due to the SU(2) symmetry, another compact group.

${ }^{*} \mathbf{3}$ One also wonders whether there might not be non-topological, instanton (finite action) solutions of the classical field equations so far overlooked by investigators searching for topological conservation laws.

\section{References}

[1] Y. Nambu, Nucl. Phys. B130 (1977) 505. 
[2] M. Jacob, ed., Dual theory (North-Holland, Amsterdam, 1974);

J. Scherk, Rev. Mod. Phys. 47 (1975) 123.

[3] See, e.g., the review of solitons by: L.D. Faddeev and V.E. Korepin, Phys. Rep., to be published.

[4] S. Coleman, Classical lumps and their quantum decedants, in: Proc. Intern. School of Subnuclear physics, Ettore Majorana (Erice, 1975).
[5] M.B. Einhorn and R. Savit, Phys. Rev. D, to be published; Fermilab-Pub-77/105 THY.

[6] R. Friedberg, T.D. Lee and A. Sirlin, Nucl. Phys. B115 (1976) $1,32$. 